\title{
Projected shell model analysis of tilted rotation
}

\author{
J. A. Sheikh, ${ }^{1,2}$ Y. Sun, ${ }^{3,4,5}$ and P. M. Walker ${ }^{1}$ \\ ${ }^{1}$ Department of Physics, University of Surrey, Surrey, Guildford, GU2 5XH, England \\ ${ }^{2}$ Tata Institute of Fundamental Research, Colaba, Bombay, 400 005, India \\ ${ }^{3}$ Joint Institute for Heavy Ion Research, Oak Ridge National Laboratory, Oak Ridge, Tennessee 37831 \\ ${ }^{4}$ Department of Physics and Astronomy, University of Tennessee, Knoxville, Tennessee 37996 \\ ${ }^{5}$ Physics Division, Oak Ridge National Laboratory, Oak Ridge, Tennessee 37831
}

(Received 15 September 1997)

\begin{abstract}
An alternative description of tilted rotation, observed in deformed nuclei, is presented using the projected shell model approach. It is shown that a strong configuration mixing among the projected states is responsible for the appearance of the tilted bands near to, but slightly above, the yrast line in even-even tungsten isotopes. Various tilted bands in ${ }^{184} \mathrm{Os}$ are also predicted. [S0556-2813(98)50701-7]

PACS number(s): 21.60.Cs, 21.10.Re, 21.60.Ev, 27.70.+q
\end{abstract}

The study of high-angular-momentum phenomena has provided a deep understanding of the motion of nucleons in a rotating mean field. It is known that this motion is strongly perturbed by the Coriolis interaction in the high- $j$ intruder orbitals and, for instance, shows up in the form of the backbending phenomenon at high angular momenta [1,2]. The conventional interpretation of the backbending is in terms of a crossing of the $s$ band with the ground-state band [3]. The $s$ band has two quasiparticles coupled to low- $K$ and orbiting around the rotational axis. (" $K$ "' is the projection of the total angular momentum along the symmetry axis). The $s$ band requires less collective rotational energy to generate angular momentum and becomes energetically favored at higher spins as compared to the ground-state band.

For a partially rotation-aligned state formed from a twoquasiparticle (2-qp) excitation, the total $K$ quantum number of the state can be obtained either by the addition or subtraction of the $\Omega$ quantum numbers of the individual quasiparticles. We thus can have two kinds of couplings: a 2-qp state with a larger total $K$ (high- $K$ ) and a state with a smaller $K$ (low- $K$ ). We can discuss two situations according to the shell filling: in the lower half of the intruder subshell, both individual quasiparticle states have small $\Omega$ numbers and the total $K$ numbers for both of the coupling schemes are relatively small. Therefore, they both can contribute to the $s$ band. However, when we move to the upper half of the subshell, both of the individual quasiparticle states have larger $\Omega$ numbers. The coupling in this case will lead to two 2-qp states with very different total $K$ values-one is small and the other is large. Usually, only the low- $K$ state is assumed to contribute to the $s$ band.

However, one should keep in mind that in the above discussion, we have assumed that the rotational axis is perpendicular to the symmetry axis. In this case, high- $K$ bands are less easy to align along the rotational axis because it requires more energy to generate angular momentum. Nevertheless, there is experimental evidence that as the Fermi surface moves into the upper half of the intruder subshell, the states with high- $K$ start emerging around the yrast line (with the lowest eigenenergy for each angular momentum) and these bands start competing with the low- $K s$ bands [4]. This has been observed in $\mathrm{W}, \mathrm{Re}$, and Os nuclei [4-8] challenging the traditional cranking picture. It seems to suggest a modification of the conventional picture that nuclei should always rotate around an axis perpendicular to the symmetry axis $[9,10]$. This led to the idea of the tilted axis cranking (TAC) approach in order to interpret $[8,11,12]$ the high- $K$ bands, subsequently called $t$ bands.

Our interest in this paper is to study the interplay between the ground-state band, the low- $K s$ band and the high- $K t$ band near the yrast line. It is observed in ${ }^{182} \mathrm{~W}$ [5] that the yrare (the first excited state for each angular momentum) band has $t$-band structure and does not change its character appreciably to high spins. However, the yrast configuration depicts a rapid change in moment of inertia and the continuation of the yrast band is not the $t$ band, despite the expectation that the $t$ band would cross the $g$ band. We would like to understand this anomalous behavior of the band crossings in this mass region and, in general, the mechanism behind the occurrence of the high- $K$ bands near the yrast line using the projected shell model (PSM) [13]. This model is ideal for studying these features since the angular-momentum projection can be performed for each $K$ configuration and the mixing among the different $K$ states can be evaluated by diagonalizing the shell model Hamiltonian in the projected basis. Bands with different $K$ configurations are calculated without assuming a semiclassical tilted axis. However, as we shall see, $t$ bands appear low in energy after angular-momentum projection and configuration mixing. The present calculations are performed for even-even tungsten isotopes, ${ }^{182,180,178} \mathrm{~W}$, which exhibit a large structural variation. ${ }^{182,180} \mathrm{~W}$ contain well-developed $t$-band structures $[5,6]$, whereas ${ }^{178} \mathrm{~W}$ shows a more regular $s$-band crossing $[14,15]$.

The PSM approach is applicable for the low- and highspin states in heavy nuclei and is based on the shell model philosophy. The PSM uses a deformed Nilsson singleparticle basis, unlike the conventional shell model which uses a spherically symmetric single-particle basis. The deformed basis has the advantage of incorporating important nuclear correlations more readily, especially for a welldeformed system. While this shell-model basis violates the rotational symmetry, it can be restored by the standard angular-momentum projection technique [16]. The pairing 
correlations are included by performing Bardeen-CooperSchreiffer (BCS) calculations with the Nilsson states. Thus, the shell-model truncation is carried out in the quasiparticle (qp) space with the qp vacuum, $|\phi\rangle$ defined through the BCS transformation. Using the PSM, it has been demonstrated that one can account quantitatively for many high-spin phenomena [13].

In this work we have used the following Hamiltonian [13]

$$
\hat{H}=\hat{H}_{0}-\frac{1}{2} \chi \sum_{\mu} \hat{Q}_{\mu}^{\dagger} \hat{Q}_{\mu}-G_{M} \hat{P}^{\dagger} \hat{P}-G_{Q} \sum_{\mu} \hat{P}_{\mu}^{\dagger} \hat{P}_{\mu}
$$

where $\hat{H}_{0}$ is the spherical single-particle energy. The second term is the quadrupole-quadrupole interaction and the last two terms are the monopole and quadrupole pairing interactions, respectively. The interaction strengths are determined as follows: the quadrupole interaction strength $\chi$ is adjusted so that the known quadrupole deformation $\varepsilon_{2}$ from the selfconsistent mean-field calculations is obtained. In our meanfield Nilsson potential, which provides the optimal deformed basis, the hexadecapole deformation has also been included. In the present calculations, we use $\varepsilon_{2}=0.225,0.232,0.240$ (with $\varepsilon_{4}=0.057,0.048,0.040$ ) for ${ }^{182} \mathrm{~W},{ }^{180} \mathrm{~W}$, and ${ }^{178} \mathrm{~W}$, respectively [17]. The monopole pairing strength $G_{M}$ is $G_{M}^{ \pm}=[21.2-13.9(N \mp Z) / A] \times A^{-1}[13]$, with " + " for neutrons and "- " for protons. The quadrupole pairing strength $G_{Q}$ is assumed to be proportional to $G_{M}$ and the proportionality constant is fixed to be 0.16 in the present work. These strengths are consistent with those used in previous works for the same mass region [13].

In the present work, we have assumed that the deformed single-particle states have axial symmetry. Thus, the basis states $\left|\varphi_{\kappa}\right\rangle$ have $K$ as a good quantum number. In the calculation, the configuration space consists of the three major shells for each kind of nucleon: $N=3,4$, and 5, for protons and $N=4,5$, and 6 for neutrons. The chosen quasiparticle subspace is spanned by the basis set

$$
\left\{|\phi\rangle, \quad \alpha_{n_{i}}^{\dagger} \alpha_{n_{j}}^{\dagger}|\phi\rangle, \quad \alpha_{p_{k}}^{\dagger} \alpha_{p_{l}}^{\dagger}|\phi\rangle, \quad \alpha_{n_{i}}^{\dagger} \alpha_{n_{j}}^{\dagger} \alpha_{p_{k}}^{\dagger} \alpha_{p_{l}}^{\dagger}|\phi\rangle\right\} .
$$

The vacuum state $|\phi\rangle$ has $K=0$, while the 2- and 4-qp states, which are typically from the intruder orbitals, can have any $K$ numbers according to coupling.

Projection of good angular momentum onto each intrinsic state generates the rotational band associated with a certain intrinsic configuration $\left|\varphi_{\kappa}\right\rangle$. For example, $\hat{P}^{I}|\phi\rangle$ will produce the ground-state band of an even-even system. The final results are obtained by, for each angular momentum $I$, solving the eigenvalue equation

$$
\sum_{\kappa^{\prime}}\left(H_{\kappa \kappa^{\prime}}-E N_{\kappa \kappa^{\prime}}\right) f_{\kappa^{\prime}}=0
$$

with the Hamiltonian and norm overlaps, $H_{\kappa \kappa^{\prime}}$ $=\left\langle\varphi_{\kappa}\left|\hat{H} \hat{P}_{K_{\kappa} K_{\kappa^{\prime}}^{\prime}}^{\mathrm{I}}\right| \varphi_{\kappa^{\prime}}\right\rangle$ and $N_{\kappa \kappa^{\prime}}=\left\langle\varphi_{\kappa}\left|\hat{P}_{K_{\kappa} K_{\kappa^{\prime}}^{\prime}}^{\mathrm{I}}\right| \varphi_{\kappa^{\prime}}\right\rangle$, respectively. The energies of each band, $E_{\kappa}(I)$, are given by the diagonal elements of $H_{\kappa \kappa} / N_{\kappa \kappa}$. A diagram in which $E_{\kappa}(I)$ for various bands is plotted against the spin $I$ will be referred to as a band diagram [13]. In a band diagram, one can see the

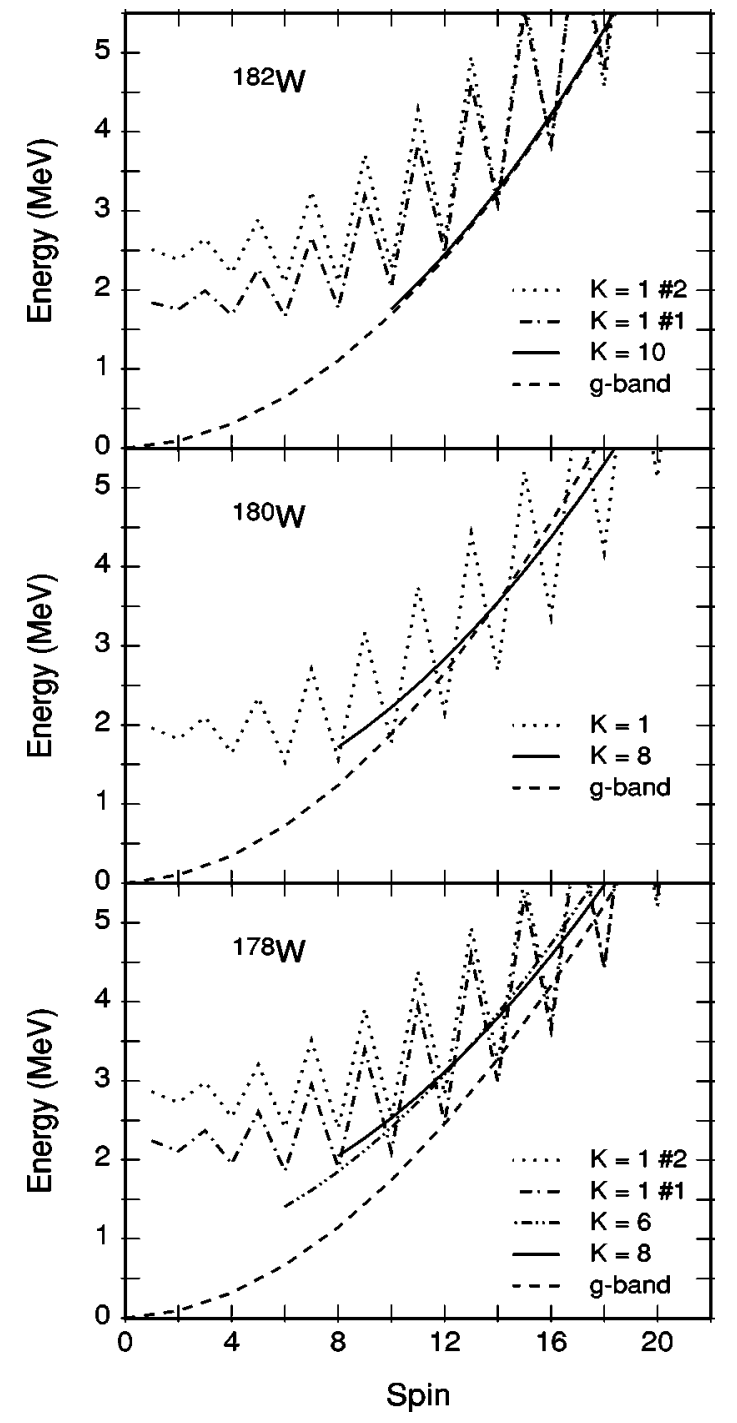

FIG. 1. Band diagrams-projected energies before configuration mixing (spins in units of $\hbar$ ). For ${ }^{182} \mathrm{~W}$, the $K=0$ and $K=1 \# 1$ band are from neutron 2-qp state [(624)9/2,(615)11/2], and the $K=1 \# 2$ band from the neutron 2-qp state $[(633) 7 / 2,(624) 9 / 2]$. For ${ }^{180} \mathrm{~W}$, the $K=8$ and $K=1$ bands consisting of the neutron 2-qp state $[(633) 7 / 2,(624) 9 / 2]$ are predicted to be around the yrast line. For ${ }^{178} \mathrm{~W}$, there are two $K=1$ bands from neutron states $[(633) 7 / 2,(624) 9 / 2](K=1 \quad \# 1)$ and $[(642) 5 / 2,(633) 7 / 2](K=1 \quad \# 2)$. The low-lying high- $K$ bands are neutron 2-qp states $[(633) 7 /$ $2,(624) 9 / 2] K=8$, and $[(512) 5 / 2,(514) 7 / 2] K=6$.

band distribution and study their properties even before the mixing. The results obtained after diagonalizing the Hamiltonian of Eq. (1), i.e., after taking their interactions into account, are to be compared with the experimental data.

The band diagrams of the PSM calculations are presented in Fig. 1 for ${ }^{182} \mathrm{~W},{ }^{180} \mathrm{~W}$, and ${ }^{178} \mathrm{~W}$ nuclei. (Note that there are typically $\sim 100$ bands to be mixed in our calculations, but we only plot a few most important ones to illustrate the physics.) In the case of ${ }^{182} \mathrm{~W}$, the ground, $K=0$ band is crossed by the $[(624) 9 / 2,(615) 11 / 2] K=1$ neutron configuration at $I=14$ and this band is then crossed by the [(633)7/2,(624)9/2] $K=1$ neutron configuration which is composed of lower- $\Omega$ states and has a larger rotational alignment. The large signature splitting for these $K=1$ bands is a 


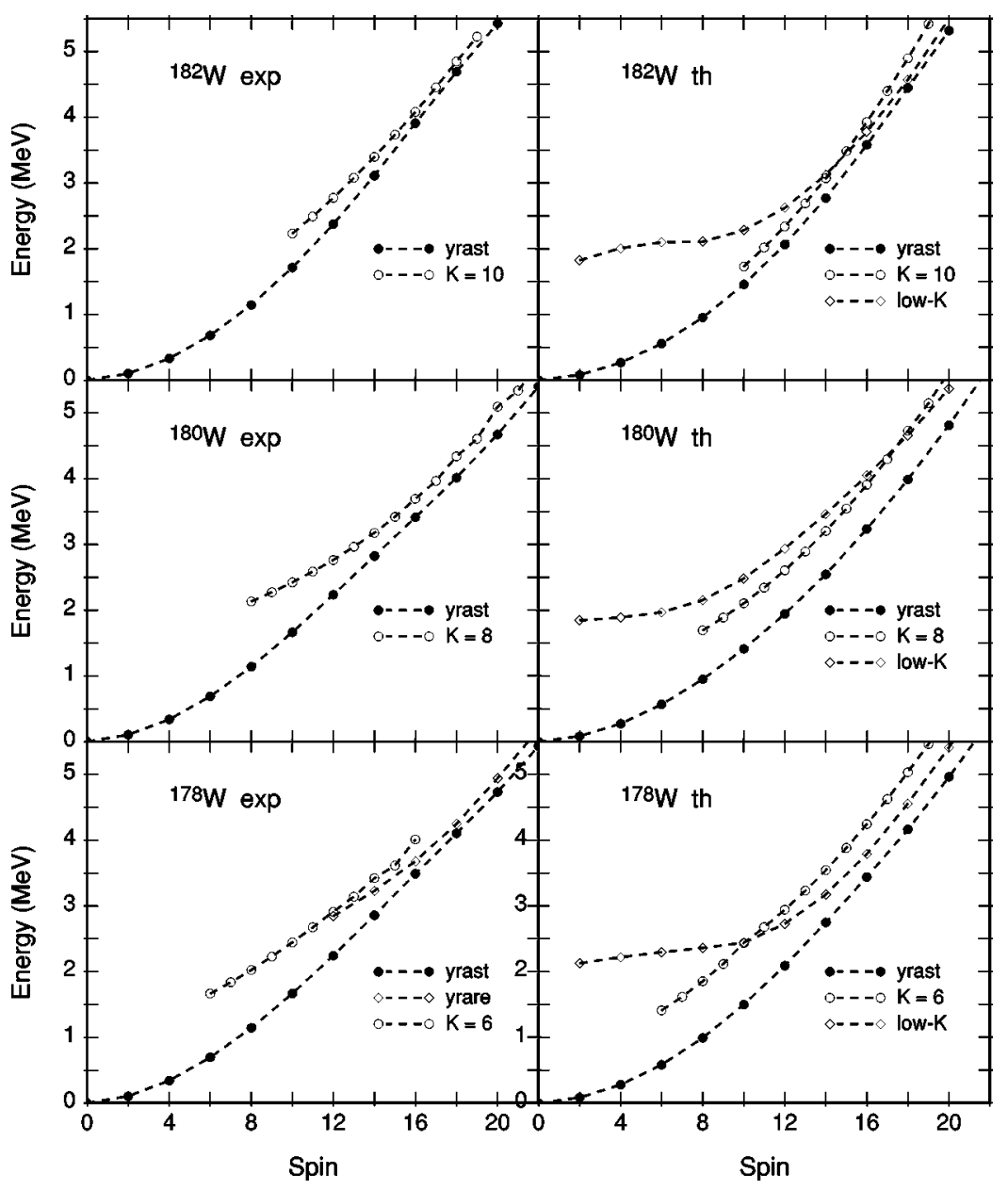

FIG. 2. Comparison of experimental data with theoretical bands obtained after configuration mixing (spins in units of $\hbar$ ). Note that the bands have mixed $K$ components and that $K$ numbers denoted here represent the dominant configuration for each band.

manifestation of strong rotational perturbation. Although, with a very large basis, the PSM results should be independent of the deformation used in the Nilsson potential, in practice a limited configuration space is employed and the results do depend on the input deformation, especially for the sensitive regions where the single-particle distribution around the Fermi surface is a strongly varying function of deformation. We do see that inclusion of a large hexadecapole deformation favors the $[7 / 2,9 / 2] K=1$ configuration. This would explain the sharp backbending observed [5] in ${ }^{182} \mathrm{~W}$. It is noted [5] that the backbending in ${ }^{182} \mathrm{~W}$ is sharper than in ${ }^{180} \mathrm{~W}$ and is somewhat puzzling since normally one would expect that, with the filling of neutrons in the higher$\Omega$ states, the backbending should be smoother. We would like to mention that the oscillatory character of the interaction strength [18] can also lead to a sharper backbend in ${ }^{182} \mathrm{~W}$.

It is noted from Fig. 1 that the $t$ band with the [(624) $9 / 2,(615) 11 / 2] K=10$ neutron configuration is almost degenerate with the ground-state $K=0$ band. The $\Omega$ decomposition of this band is the same as $[9 / 2,11 / 2] K=1$, but with the individual $\Omega$ states added up. As expected, the unperturbed bandheads $I=1$ and 10 of the two bands are almost the same, since the intrinsic Nilsson energies are identical for the two bands. The $t$ band with a large $K$-value depicts no signature splitting and the lowest odd-spin states will originate from this configuration for all spin values. As mentioned in the Introduction, these high- $K$ bands normally lie at a higher-excitation energy relative to the $K=0$ bands. How- ever, with the Fermi surface in the upper half of the intruder subshell, these $t$ bands compete with the low- $K s$ bands. Futhermore, due to the very large difference in $K$ quantum numbers, there is no term in the Hamiltonian which can directly couple the $t$ band with the $g$ and $s$ bands, thus the $t$ band remains unaffected by the configuration mixing, as discussed later.

The band diagram for ${ }^{180} \mathrm{~W}$ shows the neutron [(633)7/2,(624)9/2] $K=1$ band crossing the $K=0 \mathrm{~g}$ band at $I=10$. The tilted neutron band $[(633) 7 / 2,(624) 9 / 2] K=8$ lies higher in energy as compared to the $s$ and $g$ bands, but it will be shown later that the configuration mixing alters this ordering. The band diagram for ${ }^{178} \mathrm{~W}$ contains more structures near the yrast line. The neutron $[(633) 7 / 2,(624) 9 / 2] K=1$ band crosses the $K=0$ band at $I=12$, and this band is later (at $I=18$ ) crossed by the neutron $[(642) 5 / 2,(633) 7 / 2] K=1$ band. The $[(633) 7 / 2,(624) 9 / 2] K=8$ band lies higher in energy compared to both $g$ and $s$ bands. However, the theory predicts a low-lying $K=6$ band formed by neutrons $[(512) 5 / 2,(514) 7 / 2]$. This band lies very low at the bandhead and is crossed by the $[7 / 2,9 / 2] K=8$ band at $I=12$ with a very small crossing angle.

The PSM results after configuration mixing are compared with the experimental data $[6,5,15]$ in Fig. 2. The yrast configuration is reproduced quite well for all the three nuclei at lower spins. However, at higher spins, there is some disagreement, increasing with spin. This can be understood by noting that in our projected basis, we have configurations up to 4-quasiparticles only. The 6-quasiparticle configurations 
are known to compete in these nuclei at about the excitation energy of $\approx 4.5 \mathrm{MeV}$ and the PSM results appear to deviate above this energy.

In the case of ${ }^{182} \mathrm{~W}$, the yrast states up to $I=10$ are dominated by the $K=0$ configuration. For $I=12$, there is almost equal mixing of $[9 / 2,11 / 2] K=1$ and $K=0$ states, and for $I=14$ and 16 the contribution of $K=1$ is larger than $K=0$. The yrast $I=18$ and 20 states are predominantly composed of $[7 / 2,9 / 2] K=1$. The yrare $I=10$ to 14 states have $[9 / 2,11 / 2] K=10$ composition but then change character at higher spins. In Fig. 2, we have followed each band with a predominant intrinsic configuration.

The $t$ bands in Fig. 2 have almost no overlap with the $g$ and $s$ bands. There is no direct mixing between these bands with the $Q Q$ interaction employed in the present work. Therefore, the backbending observed in the high-spin data of ${ }^{182} \mathrm{~W}$ is due to crossing of the $[9 / 2,11 / 2] K=1$ and $[7 / 2,9 / 2] K=1 s$ bands with the ground-state band. The yrast states above $I=14$ originate from these $K=1$ configurations. In the conventional picture the yrare states above the crossing region are comprised of the $K=0 \mathrm{~g}$-band configuration. However, in Fig. 2 it is observed that the yrare states up to $I=14$ come from the $t$ band, which is due to strong configuration mixing (repelling each other) between the $g$ and the $s$ bands. In the unperturbed picture in Fig. 1, the yrare $I=14$ state has $K=0 \mathrm{~g}$-band character. The interaction pushes up the $g$ band and makes it possible for the $t$ band to form the yrare band.

For ${ }^{180} \mathrm{~W}$, the yrast states up to $I=8$ are mainly comprised of the $K=0$ configuration. For $I=10$, the yrast state has a large $[7 / 2,9 / 2] K=1$ contribution and this increases with increasing angular momentum. The yrare even-spin states up to $I=16$ and all the yrast odd-spin states have mainly $[7 / 2,9 / 2] K=8$ composition. It is to be noted that before the configuration mixing, the yrare states belong to $[7 / 2,9 / 2] K=1$. However, the mixing between the $g$ and the $[7 / 2,9 / 2] K=1$ bands is even stronger than that in ${ }^{182} \mathrm{~W}$ and this pushes the $[7 / 2,9 / 2] K=1, I=8$ state to higher energy. The yrare states from $I=8$ to 16 have mainly $[7 / 2,9 / 2] K=8$ composition. The $I=18,20$, and 22 yrare states have a strong mixing from the $K=1$ state and the even-spin states above are mainly composed of this configuration. At present, our calculation fails to reproduce the magnitude of the observed staggering in the yrare band between the even and odd higher spin states. Qualitatively, this staggering could be understood as a consequence of interaction of the $K=8$ band with other low- $K$ bands (e.g., the $g$ band): the even-spin states of the $K=8$ band are pushed up by the interaction, while the odd spin states remain unperturbed (there is no odd spin state from the $g$ band to interact with). This question has been addressed in a model calculation in Ref. [19]. To further expose the deviation, a comparison of the staggering part of the $K=8$ band in ${ }^{180} \mathrm{~W}$ for our calculation with the data is shown in Fig. 3, for quantity of $\frac{1}{2}[E(I+1)$ $-E(I-1)]$. It is seen that our calculations produce the right staggering phase, but the amplitude is too small as compared to the data. In addition, the theoretical results underestimate the alignment of the $K=8$ band.

The even-spin yrast states for ${ }^{178} \mathrm{~W}$ up to $I=8$ mainly arise from the $K=0 \mathrm{~g}$-band configuration, from $I=8$ to $I=$

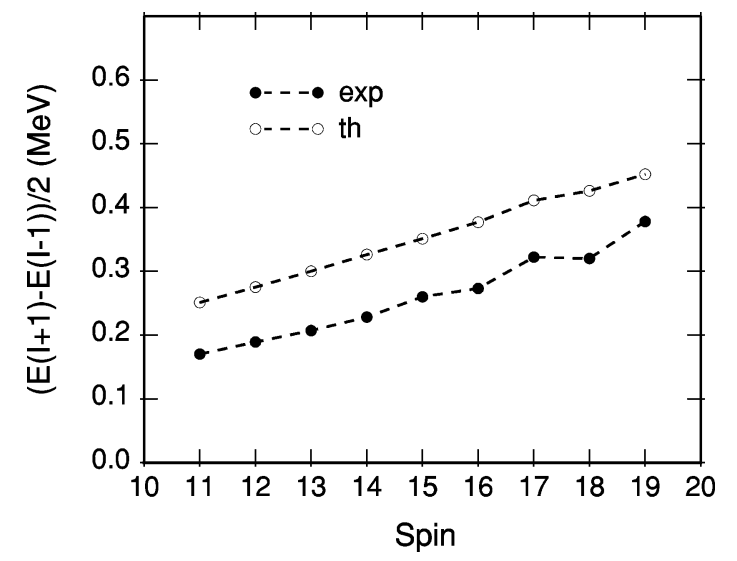

FIG. 3. Comparison for the staggering part of the $K=8$ band in ${ }^{180} \mathrm{~W}$ for our calculation with the data (spins in units of $\hbar$ ). The quantity to be compared is $\frac{1}{2}[E(I+1)-E(I-1)]$.

14 they are a mixture of $K=0$ and [(633)7/2,(624)9/2] $K=1$ states and from $I=16$ and above they are highly mixed and arise from $[(633) 7 / 2,(624) 9 / 2] K=1$ and $[(642) 5 / 2,(633) 7 / 2] K=1$. The yrare states up to $I=10$ orginate from the $[(512) 5 / 2,(514) 7 / 2] K=6$ neutron configuration. If we follow this high- $K$ configuration, it has a strong mixture with the neutron $[(633) 7 / 2,(624) 9 / 2] K=8$ $t$-band structure before and after their band crossing (see Fig. 1 ), and it eventually becomes dominated by the $[7 / 2,9 / 2] K=8$ component. However, the yrare structure is changed to the superpositions of $K=0,[7 / 2,9 / 2] K=1$ and $[5 / 2,7 / 2] K=1$ configurations after $I=12$, as we clearly see in Fig. 2. We would like to mention that the data [15] also show a dipole band with bandhead spin $I=6$ and a clear crossing of two bands at $I=12$.

Our discussion of $t$ bands has so far been concentrated on neutron configurations. By adding two protons, i.e., going up to the osmium isotopes, the proton Fermi level also lies between high- $K$ orbitals with $K=9 / 2$ and $11 / 2$. It is thus very interesting to see how the proton 2-qp high- $K$ band competes with the neutron high- $K$ bands discussed earlier. Bands for ${ }^{184}$ Os are calculated with the same parameters as for the tungsten isotopes except for the deformation $\left(\varepsilon_{2}=0.210\right.$ and $\varepsilon_{4}=0.053$ [17]). In Fig. 4, the band diagram is plotted in the upper half, while results after band mixing are given in the lower part. Indeed, the proton $[(514) 9 / 2,(505) 11 / 2] K=10$ band is found lower than the neutron [(624)9/2,(615)11/2]K $=10$ band, and is nearly degenerate with the $g$ band. Although ${ }^{184}$ Os has the same neutron number as ${ }^{182} \mathrm{~W}$, due to smaller deformation the neutron $[(633) 7 / 2,(624) 9 / 2] K=8$ band is also predicted lying low and can compete with the neutron $K=10$ band. In addition, a $K=114$-qp band, being a combination of the proton $K=10$ and neutron $K=1$ structure, sharply dives down at high spins. For these 6 lowest bands, besides the $g$ band with a neutron $K=1$ band crossing, we may focus on another two interesting bandcrossings, i.e., the crossing between the neutron $K=8$ and $K=10$ bands at $I=12$ and the one between the proton $K=10$ and the $K=114$-qp band at $I=15$. After band mixing, the partners are pushed up or down. The neutron $K=10$ band is strongly mixed with the $K=8$ band at the crossing region and has more $K=8$ component afterwards. The proton $K=10$ band is the yrare band 


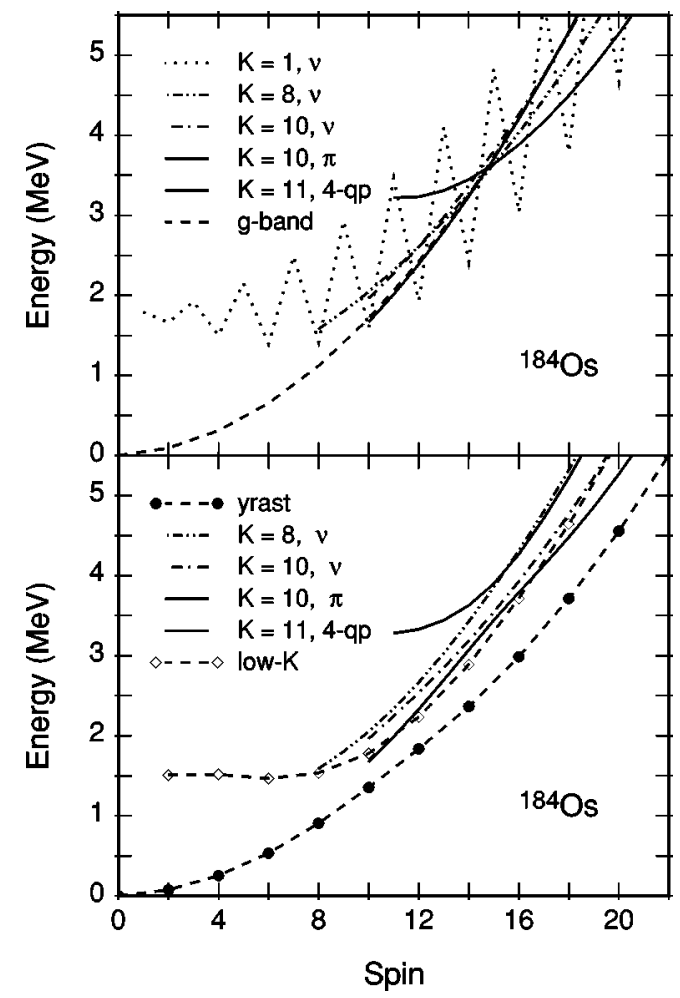

FIG. 4. Predictions for ${ }^{184}$ Os. The upper panel shows the band diagram and the lower panel contains the results after configuration mixing (spin in units of $\hbar$ ). In the lower panel, the $K=8$ and 10 neutron $t$ bands are both shown by dashed-dotted lines since they interact strongly. The two-quasiproton band with $K=10$ and the four-quasiparticle band with $K=11$ also interact strongly and both are shown by full lines.

around the bandhead spin. By the strong interaction with the 4-qp band, it is pushed down at $I=15$ and becomes the yrare band again. Actually, this band becomes dominated by the 4 -qp component after $I=15$. It would be interesting to observe these predicted phenomena in a future experiment.
A question that remains to be explored adequately is the degree of mixing of the high- $K t$ configuration into the yrast band. This has potentially important consequences for the understanding of anomalous $K$-isomer decays by highly $K$-forbidden transitions [4]. However, the extent of the $t$ band/yrast band mixing, here found to be very small, is sensitive to the model space and the Hamiltonian employed. While the effect on level energies may not be significant, the evaluation of transition matrix elements requires further work.

In conclusion, we have studied the near-yrast band structures in tungsten isotopes with focus on the tilted bands that become energetically favored with the Fermi surface in the upper half of the intruder subshell. We have demonstrated how the tilted bands can appear at low energy by angular momentum projection and configuration mixing, within a model going beyond the usual mean-field approach. The observed backbending in these nuclei comes from the normal $s$ band crossing. However, due to a strong interaction between the $g$ and $s$ bands, the two bands repel after the configuration mixing and the normal yrare band lies at a higher energy. In constrast, the $t$ band has almost no coupling with these bands and remains largely unaffected after the mixing. This explains the observation of the $t$ band as the yrare band in the experimental data of ${ }^{180,182}$ W. Finally, a complex and interesting structure is predicted for ${ }^{184} \mathrm{Os}$.

The Joint Institute for Heavy Ion Research has as member institutions, the University of Tennessee, Vanderbilt University, and the Oak Ridge National Laboratory; it is supported by the member institutions and by the U.S. Department of Energy through Contract No. DE-AS05-76ER04936 with the University of Tennessee. Oak Ridge National Laboratory is managed by Lockheed Martin Energy Research Corporation for the U.S. Department of Energy under Contract No. DE-AC05-96OR22464. Support has also been received from the U.K. EPSRC under Grant No. GR/K84219.
[1] A. Johnson, H. Ryde, and J. Sztarkier, Phys. Lett. 34B, 605 (1971).

[2] A. Johnson, H. Ryde, and S.A. Hjorth, Nucl. Phys. A179, 753 (1972).

[3] F.S. Stephens and R.S. Simon, Nucl. Phys. A183, 257 (1972).

[4] P.M. Walker et al., Phys. Rev. Lett. 67, 433 (1991).

[5] T. Shizuma et al., Nucl. Phys. A593, 247 (1995).

[6] P.M. Walker et al., Phys. Lett. B 309, 17 (1993).

[7] T. Kutsarova et al., Nucl. Phys. A587, 111 (1995).

[8] C.J. Pearson et al., Phys. Rev. Lett. 79, 605 (1997).

[9] P. Ring and H.-J. Mang, Phys. Rev. Lett. 33, 1174 (1974).

[10] R. Bengtsson and S. Frauendorf, Nucl. Phys. A327, 139 (1979).
[11] S. Frauendorf, Nucl. Phys. A557, 259c (1993).

[12] J.A. Sheikh, Phys. Rev. C 52, 3061 (1995).

[13] K. Hara and Y. Sun, Int. J. Mod. Phys. E 4, 637 (1995).

[14] C. Dors et al., Nucl. Phys. A314, 61 (1979).

[15] C.S. Purry et al., Phys. Rev. Lett. 75, 406 (1995); Nucl. Phys. A (in press).

[16] P. Ring and P. Schuck, The Nuclear Many Body Problem (Springer-Verlag, New York, 1980).

[17] R. Bengtsson, S. Frauendorf, and F.-R. May, At. Data Nucl. Data Tables 35, 15 (1986).

[18] R. Bengtsson, I. Hamamoto, and B.R. Mottelson, Phys. Lett. 73B, 259 (1978).

[19] S. Frauendorf and J. Meng, Z. Phys. A 356, 263 (1996). 